\title{
Statistical Analysis on Preliminary Breakdown Pulses of Positive Cloud-to-Ground Lightning in Malaysia
}

\author{
Chin-Leong Wooi, Zulkurnain Abdul-Malek, Noor Azlinda Ahmad, Mehrdad Mokhtari, \\ Behnam Salimi \\ Institute of High Voltage and High Current, Faculty of Electrical Engineering, Universiti Teknologi Malaysia,
} Johor Bahru, Malaysia

\begin{tabular}{l} 
Article Info \\
\hline Article history: \\
Received Oct 10, 2015 \\
Revised Dec 14, 2015 \\
Accepted Jan 6, 2016 \\
\hline
\end{tabular}

Keyword:

Cloud-to-ground

Lightning discharge

Positive lightning

Preliminary breakdown pulses

\begin{abstract}
This aim of this paper is to study the preliminary breakdown pulses (PBP) of positive cloud-to-ground $(+\mathrm{CG})$ lightning flashes in Malaysia. There is a difference in PBP characteristics when a comparison is made between positive and negative flashes. To the best of the authors' knowledge, this is the first time the PBP characteristics preceding positive lightning in Malaysia are reported. This study was carried out in Johor Bahru, Malaysia using a broadband antenna system during a monsoon period (Oct-Dec, 2012). It is found that the PBP for $+\mathrm{CG}$ lightning is much more complicated compared to the positive cloud-to-ground $(-\mathrm{CG})$ lightning since only negative PBP trains are usually present in the $-\mathrm{CG}$ lightning. Out of 21 analyzed positive flashes, four types of PBP trains were identified according to their initial polarities. These are negative PBP (20\%), positive PBP (15\%), PBP with polarity reversal $(10 \%)$, and PBP with irregular polarity $(15 \%)$. The balance $40 \%$ of flashes is without any PBP. The effect of geographical region may also cause the observed differences in the cloud discharge processes including the types of PBP. More studies are needed to determine the relationship between the return stroke and PBP.
\end{abstract}

Copyright () 2016 Institute of Advanced Engineering and Science. All rights reserved.

\section{Corresponding Author:}

Zulkurnain Abdul-Malek, Institute of High Voltage and High Current

Faculty of Electrical Engineering,

Universiti Teknologi Malaysia, Johor Bahru, Malaysia

Email: zulkurnain@utm.my

\section{INTRODUCTION}

Lightning is one of the natural disaster that caused many power failure world wide [1]. A negative ground lightning flash is usually initiated at the lower edge of a negative charge center, and the breakdown process is aided by the electric field enhancement caused by a positive charge pocket located below the negative charge center known as the preliminary breakdown pulses (PBP) or initial breakdown pulses [2]. The features of PBP trains in negative cloud-to-ground (CG) flashes have been previously reported [3-14]. The return stroke of positive lightning is also sometimes preceded by a train of bipolar pulses (or PBP) lasting in a duration of few millisecond [15]. These pulse trains may provide information concerning the first event that leads to the electrical breakdown in the cloud. However, the mechanism for preliminary breakdown prior to positive lightning has yet to be confirmed $[6,16]$. The largest amplitude of PBP is usually much smaller than that of the following return stroke, but sometimes it can be comparable or even larger than that of the return stroke [8]. The time interval between PBP and the first return stroke is in the order of $10 \mathrm{~ms}$, with a maximum and minimum of several hundred milliseconds $[4,17,18]$ and few millisecond [5, 18, 19], respectively. The percentage of CG lightning with discernable PBP varies significantly in different studies. As summarized by [20], such percentage apparently varies with latitude. The smallest percentage is $19 \%$, 
recorded in Sri Lanka $\left(7^{\circ} \mathrm{N}\right)$. In regions of higher latitudes, the percentage is much higher, such as $80 \%$ in Austria, 90\% in Finland, and 100\% in Sweden.

Despite the fact that many counties already reported the PBP preceding positive return stroke $[4,6$, $16,21,22]$, apart from our own study reported in [23], this is the first time preliminary breakdown preceding positive lightning in Malaysia is reported. The initial polarity of PBP in negative cloud-to-ground (-CG) lightning is always the same as that of the first return stroke. However, the situation for positive cloud-toground $(+\mathrm{CG})$ lightning is much more complicated. Opposite initial polarities between PBP and the following return stroke are occasionally observed $[16,19]$. In this study, positive lightning shows both return stroke cases, eitherwith or without the preceded PBP. Based on these findings, PBP in positive CG lightning was classified into four types which includes the negative PBP (-PBP), positive PBP (+PBP), PBP with polarity reversal, and PBP with irregular polarity. Furthermore, the data available in the literature on PBP of positive lightning from tropical thunderclouds is scarce, and there is a need to gather more data from tropical thunderclouds. This paper aims to investigate the nature and temporal characteristic of the PBP trains in tropical regions. This study was carried out in Johor Bahru, Malaysia using a broadband antenna system during the monsoon period (Oct-Dec, 2012). Interestingly, any variation in the PBP trains for geographical regions may indicate differences in the initial breakdown processes in the clouds. Therefore, it is important to analyze and compare the features of these pulse trains in different geographical regions.

\section{RESEARCH METHOD}

The lightning electric field measurements were performed within the monsoon period (Oct-Dec, 2012) at the Observatory, Universiti Teknologi Malaysia (UTM), Johor. UTM is located in the southern portion of Peninsular Malaysia which located in tropical regions. The Observatory building is located at 142 $\mathrm{m}$ above the sea level. The configuration of the measuring system is shown in Figure 1. The setup is similar to experimental setups used in [24-27]. The vertical component of electric field was captured using $1.5 \mathrm{~m}$ height flat plate antenna. The antenna was connected to a digital oscilloscope (LeCroy Wave Runner 44Xi-A, 8-bit resolution with a sampling rate of $50 \mathrm{MSs}^{-1}$ ) via an electronic buffer circuit. The buffer acts to limit overvoltages and as an impedance matching between the antenna and oscilloscope with a frequency range of $\mathrm{kHz}$ to GHz. The buffer circuit consists of an MSK0033 buffer amplifier, capacitor and resistors. The input impedance of MSK0033 is $10^{12} \Omega$. RG58 coaxial cables were used in all connections except for the DSO-PC connection. The oscilloscope was set $150 \mathrm{~ms}$ pre-trigger mode. The decaying time constant of the buffer was set to approximately $10 \mathrm{~ms}$ by changing the RC configuration inside the buffer. Criteria to identify the PBP are similar to $[9,10,28]$ which mentioned that only pulses with amplitude larger than twice of noise level are included. Atmospheric sign convention is used throughout the paper thus a positive return stroke $(+\mathrm{CG})$ produces initially negative field changes.

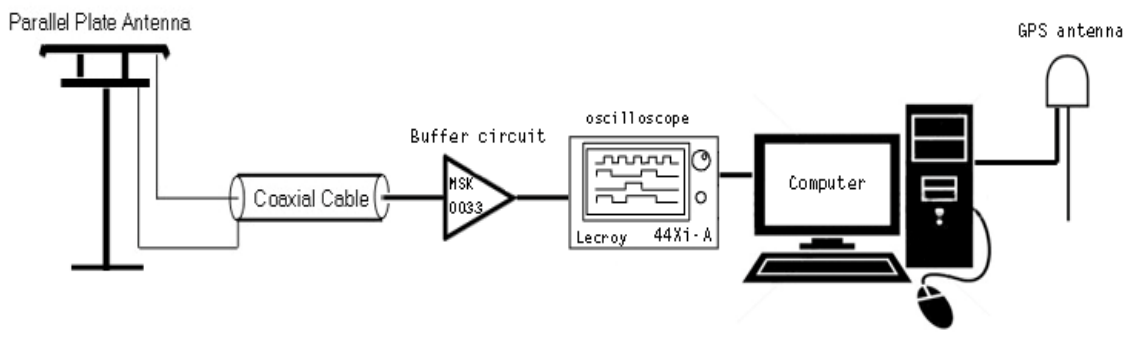

Figure 1. Schematic description of the lightning detection system

\section{RESULTS AND ANALYSIS}

It is common to notice that the initial polarity of PBP for a $-\mathrm{CG}$ flash is always negative, that is the same polarity as the negative return stroke. The BIL model was first proposed by [3], in which the return stroke is initiated by three sequential sections. The initial breakdown (B), or PBP, is the first step which lasts for several milliseconds, and it contains intense electromagnetic radiation. This breakdown is followed by an intermediate state (I) with little electric field change. Finally, a leader change (L) follows the intermediate state, and this is consequently followed by a return stroke. 


\subsection{BIL Model in Positive Lightning}

The BIL model of $+\mathrm{CG}$ was found to be similar with BIL model in -CG [21]. However, the situation for $+\mathrm{CG}$ lightning is much more complicated. Opposite initial polarities between PBP and the following return stroke are occasionally observed $[14,16]$.Waveforms of different types of PBP are generally similar with each other. As can be seen in Figure 2, each pulse train usually starts with one or two very small pulses, followed by some largest pulses of the train, and ends with pulses of decreasing amplitudes.

Pulses in PBP train are mainly bipolar. Pulses at the beginning and middle of the train usually have a very clear bipolar feature. However, at the end of the train, pulses sometimes appear as unipolar. Such an example is given in Figure 2. It can be seen that before the time of about $-22.35 \mathrm{~ms}$, pulses are clearly bipolar, but during the time of $-21 \mathrm{~ms}$ to $-20 \mathrm{~ms}$, pulses seem to be unipolar, and it is difficult to tell whether they belong to PBP or the intermediate state. The intermediate stage in the BIL model of PBP is thought to be caused by horizontal propagation of leader channel [21]. Such horizontal propagation produces little vertical electric field changes at the ground and makes the time interval between PBP and the first return stroke longer.

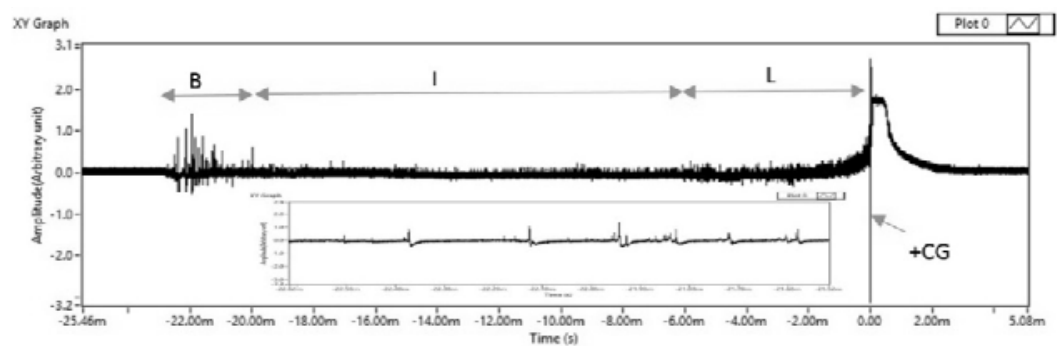

Figure 2. Flash No. 271112.219 The sample waveform with negative initial polarity during + CG compatible with BIL model

\subsection{BL Model in Positive Lightning}

Although the BIL model is useful as a general description for the lightning characterization, the manner of the breakdown phenomenon differs in the thunderstorms. The behavior of the lightning flashes varies from study to study, so it is not completely standardized. The existence of some PBP which is followed directly by small pulses is possibly an indication of leader changes and then followed by the return stroke without the intermediate stage named as BL model. The BL model of $+\mathrm{CG}$ has been shown in Figure 3. One reason for the disparity between these two model of PBP in $+\mathrm{CG}$ may be due to the different distances from observing site, as in large distance, small signals produced by stepped leaders may be not detected, resulting in an intermediate stage. However, Ting $\mathrm{Wu}$ et al. reported that the differences between these two model of PBP may be due to different physical processes of preliminary breakdown or stepped leader [21].

Sometimes, it is difficult to differentiate the B, and L parts, as pulses at the end of the train seem to gradually change from bipolar to unipolar. Such an example is given in Figure 3. It can be seen that before about $-10 \mathrm{~ms}$, pulses are clearly bipolar, but during $-10 \mathrm{~ms}$ to $-10.50 \mathrm{~ms}$ time, pulses seem to be unipolar, and it is difficult to tell whether they belong to PBP or stepped leader pulses. In such cases, defining the end of PBP train is somehow subjective, and it also may have up to $0.5 \mathrm{~ms}$ error in calculating the duration of PBP train.

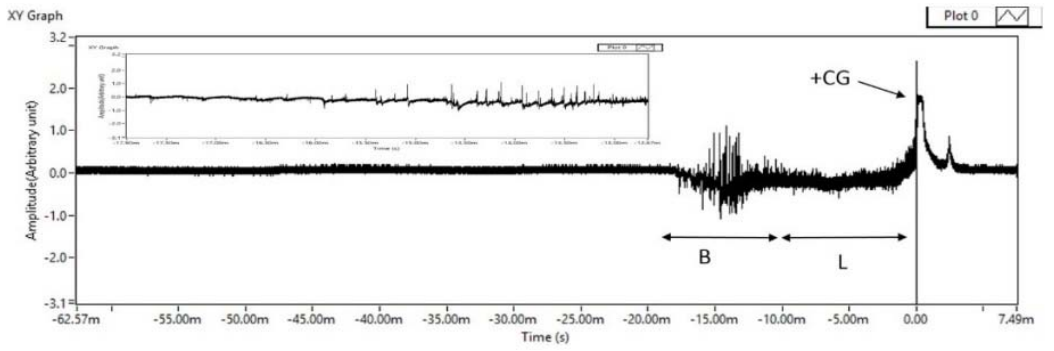

Figure 3. Flash No. 271112.209 The sample waveform with positive and negative initial polarity during $+\mathrm{CG}$ compatible with BL model 


\subsection{Types of PBP Detected in Positive Cloud-to-Ground Lightning}

Other than BIL and BL models, opposite initial polarities between PBP and the following return stroke are occasionally observed $[16,19]$. Therefore, $\mathrm{PBP}$ of $+\mathrm{CG}$ is classified into four types according to its initial polarities which are $-\mathrm{PBP}(20 \%),+\mathrm{PBP}(15 \%)$, PBP with polarity reversal $(10 \%)$, and PBP with irregular polarity (15\%). Out of the 20 flashes, 8 flashes occurred without any PBP (40\%). Two PBP trains had PBP with polarity reversal. The first region might consists of pulses with positive initial polarity and the second with pulses of negative initial polarity and vice versa. Two PBP trains contain pure positive initial pulse (+PBP) and four PBP trains contains pure negative initial pulse (-PBP). There are only 3 cases that PBP trains consist pulses with irregular polarity. The summary of types of PBP detected in positive lightning in relation with BIL and BL model is as shown in Table 1.

Table 1. Types of PBP detected in positive lightning in relation with BIL and BL model

\begin{tabular}{cccccc}
\hline Type of PBP & -PBP & + PBP & $\begin{array}{c}\text { PBP with } \\
\text { polarity reversal }\end{array}$ & $\begin{array}{c}\text { PBP with } \\
\text { irregular polarity }\end{array}$ & Without PBP \\
\hline BIL & 2 & 2 & - & 2 & - \\
BL & 2 & 1 & 2 & - & - \\
Total & 4 & 3 & 2 & 3 & 8 \\
\hline
\end{tabular}

Figure 2 shows a -PBP trains which is one of the most common pulse trains that precede $+\mathrm{CG}$ flashes. This PBP contained pulse with negative initial polarity. It is noticed that from Figure 2 and 3, if PBP is very strong, the following first return stroke always happenaftera short time; on the other hand, if there is a long wait between PBP and the first return stroke, the PBP may be weak compared with the return stroke (RS). As it can be seen in Figure 2, the leading edge of the initial half cycle of the -PBP is relatively smooth compared to that of the pulses in the other types of PBP trains. Figure 3 shows that PBP trains had two distinct regions. The first region has pulses of positive initial polarity and the second region has pulses of negative initial polarity. The two regions with pulses of opposite polarity are given in an expanded time scale. Pulses in the first region are very similar in profile to those of narrow bipolar pulse trains. The pulse profile of the second region is somewhat similar to that of -PBP trains in Figure 2. This Figure shows that the magnitude of the pulses in the second region is larger than that in the first region.

An irregular initial polarity in positive cloud-to-ground lightning is shown in Figure 4.

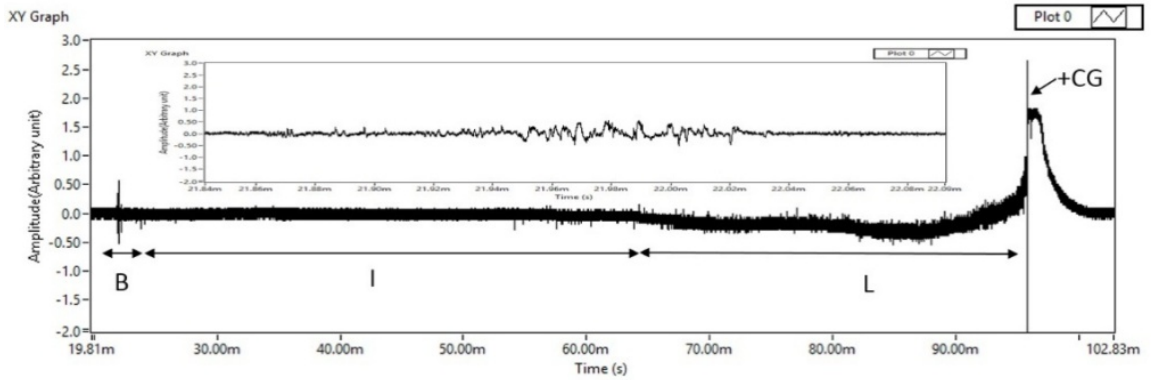

Figure 4. Flash No. 271112.216 The sample waveform with irregular initial polarity during +CG enlarge compatible with BIL model

Some irregular pulse activity is visible for a few milliseconds, even after the return stroke. However, [29] has observed that in a number of positive return strokes, a pulse burst appeared immediately after the return stroke, in which case, there was no pulse activity immediately prior to the return stroke. Hence, one cannot confidently conclude, that the pulses, which appear after the return stroke are continuations of the respective PBP trains. The duration from the beginning of PBP trains to return stroke in Figure 4 is $73 \mathrm{~ms}$ and the mean width of the pulses is $4.5 \mu \mathrm{s}$. The mean pulse separation is $11 \mu \mathrm{s}$. It seems that this PBP trains can be considered to be different from chaotic pulse trains that occur mainly prior to the negative return stroke.

Figure 5 shows the $+\mathrm{PBP}$ trains occurred before $+\mathrm{CG}$. The $+\mathrm{PBP}$ and $-\mathrm{PBP}$ in $+\mathrm{CG}$ have almost the same characteristics except of their initial polarity, and it is speculated that different initial polarities are caused by different directions of channel propagation. The PBP-RS ratio for BIL and BL model + CG shown 
in figures 4 and 3 are 0.21 and 0.42, while the PBP-RS ratio for -PBP and +PBP in Figure 2 and 5 are 0.43 and 0.59.This had shown that the peak value of RS is stronger than PBP.

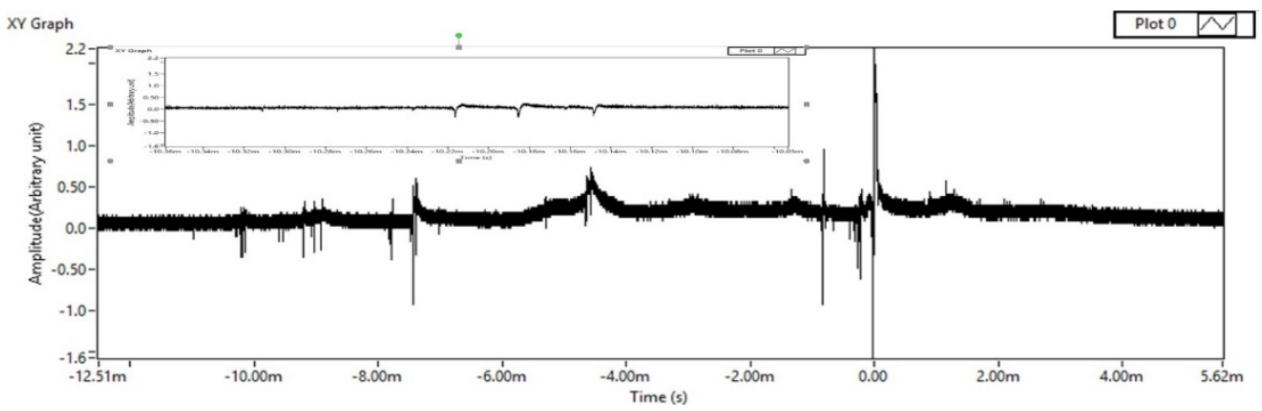

Figure 5. Flash No. 271112.254 The sample waveform with positive initial polarity during +CG

The absence of preliminary breakdown pulses in positive cloud-to-ground lightning was also found. This is shown in Figure 6. The oscilloscope was set to save the signals $150 \mathrm{~ms}$ before the triggering time to capture the whole PBP trains. As it is clear in Figure 6 the first return stroke happened at 150ms after the trigger time. However, there is no detectable PBPs before the return stroke. The return stroke happens with no significant electric field change other than that of small stepped leader pulse found prior to the return stroke.

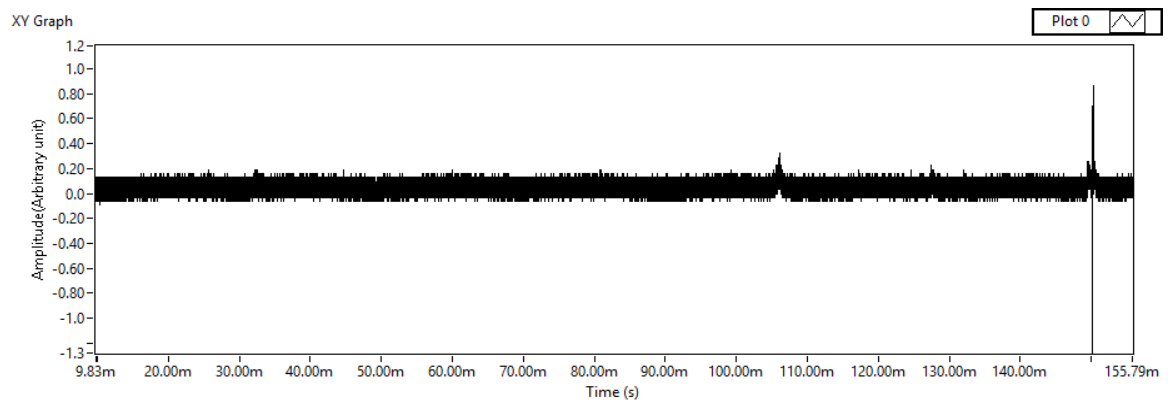

Figure 6. Flash No. 281112.164 The sample waveform without PBPs trains during +CG

\section{CONCLUSION}

Both BIL and BL models of PBP were found in $+\mathrm{CG}$ and $-\mathrm{CG}$. However, the characteristic of PBP in positive lightning was found to be different with those in negative lightning. According to their initial polarities, the PBP in positive CG lightning is classified into four typeswhich are-PBP, +PBP, PBP with polarity reversal, and PBP with irregular polarity. Out of the 20 flashes, 8 flashes, or $40 \%$ of flashes, occurred without any PBP. It is noticed that if PBP is very strong, the duration between PBP and RS is short.On the other hand, if there is a long wait between PBP and the first return stroke, the PBP is always weak compared with the RS. The PBP-RS ratio for BIL and BL models are 0.21 and 0.42 , respectively, while the PBP-RS ratio for -PBP and + PBP are 0.43 and 0.59 , respectively. For two distinct regions of PBP, results show that the magnitude of the pulses in the second region, which has a negative polarity, is larger than that in the first region (positive polarity). Several positive lightning flashes occurred without any PBP process. The effect of geographical region may also cause the observed differences in the cloud discharge processes including the types of PBP. More studies are needed to determine the relationship between the return stroke and PBP.

\section{ACKNOWLEDGEMENTS}

Authors wish to thank Universiti Malaysia Perlis (Unimap), Ministry of Education (MOE), and Universiti Teknologi Malaysia (Research Vote No. 4F291 and 10H61) for the financial aid. 


\section{REFERENCES}

[1] T.H. Zeng, et al., "Study on Lightning Intruding Overvoltage in Yantan Extension Substation," TELKOMNIKA Indonesian Journal of Electrical Engineering, vol. 12, pp. 2458-2464, 2014.

[2] M.J. Murphy, et al., "Lightning charge analyses in small Convection and Precipitation Electrification (CaPE) experiment storms," Journal of Geophysical Research: Atmospheres (1984-2012), vol. 101, pp. 29615-29626, 1996.

[3] N. Clarence and D. Malan, "Preliminary discharge processes in lightning flashes to ground," Quarterly Journal of the Royal Meteorological Society, vol. 83, pp. 161-172, 1957.

[4] W. Beasley, et al., "Electric fields preceding cloud-to-ground lightning flashes," Journal of Geophysical Research: Oceans (1978-2012), vol. 87, pp. 4883-4902, 1982.

[5] M. Brook, "Breakdown electric fields in winter storms," Res. Lett. Atmos. Electr, vol. 12, pp. 47-52, 1992.

[6] C. Gomes and V. Cooray, "Radiation field pulses associated with the initiation of positive cloud to ground lightning flashes," Journal of atmospheric and solar-terrestrial physics, vol. 66, pp. 1047-1055, 2004.

[7] J.S. Mäkelä, et al., "Properties of preliminary breakdown processes in Scandinavian lightning," Journal of Atmospheric and Solar-Terrestrial Physics, vol. 70, pp. 2041-2052, 2008.

[8] A. Nag and V.A. Rakov, "Electric field pulse trains occurring prior to the first stroke in negative cloud-to-ground lightning," Electromagnetic Compatibility, IEEE Transactions on, vol. 51, pp. 147-150, 2009.

[9] Z.A. Baharudin, et al., "Comparative study on preliminary breakdown pulse trains observed in Johor, Malaysia and Florida, USA," Atmospheric research, vol. 117, pp. 111-121, 2012.

[10] Z.A. Baharudin, et al., "Electric field changes generated by the preliminary breakdown for the negative cloud-toground lightning flashes in Malaysia and Sweden," Journal of Atmospheric and Solar-Terrestrial Physics, vol. 84, pp. $15-24,2012$.

[11] K. Mehranzamir, et al., "Comparative study of lightning models with lightning discharges in Malaysia," in Electrical Insulation and Dielectric Phenomena (CEIDP), 2013 IEEE Conference on, pp. 1085-1088, 2013.

[12] B. Salimi, et al., "Statistical Analysis of Lightning Electric Field Measured Under Equatorial Region Condition," Procedia Technology, vol. 11, pp. 525-531, 2013.

[13] Z.A.M.C.L. Wooi, Noor Azlinda Ahmad, Mehrdad Mokhtari and Amir Hesam Khavaridsa, "A Review of Recent Studies on Positive Lightning," Applied Mechanics and Materials, In press.

[14] Q. Li, et al., "Transform of Lightning Electromagnetic Pulses Based on Laplace Wavelet," TELKOMNIKA Indonesian Journal of Electrical Engineering, vol. 11, pp. 4911-4917, 2013.

[15] C. Schumann, et al., "Electric fields changes produced by positives cloud-to-ground lightning flashes," Journal of Atmospheric and Solar-Terrestrial Physics, vol. 92, pp. 37-42, 2013.

[16] A. Nag and V.A. Rakov, "Positive lightning: An overview, new observations, and inferences," Journal of Geophysical Research: Atmospheres (1984-2012), vol. 117, p. D08109, 2012.

[17] E. Pierce, "Electrostatic field-changes due to lightning discharges," Quarterly Journal of the Royal Meteorological Society, vol. 81, pp. 211-228, 1955.

[18] Z.A. Baharudin, et al., "Electric field changes generated by preliminary breakdown pulse for positive lightning ground flashes in Sweden."

[19] T.O. Ushio, et al., "Electric fields of initial breakdown in positive ground flash," Journal of Geophysical Research: Atmospheres (1984-2012), vol. 103, pp. 14135-14139, 1998.

[20] A. Nag and V.A. Rakov, "Some inferences on the role of lower positive charge region in facilitating different types of lightning," Geophysical Research Letters, vol. 36, 2009.

[21] T. Wu, et al., "Preliminary breakdown pulses of cloud-to-ground lightning in winter thunderstorms in Japan," Journal of Atmospheric and Solar-Terrestrial Physics, vol. 102, pp. 91-98, 2013.

[22] Z.A. Baharudin, et al., "Electric field changes generated by preliminary breakdown pulse for positive lightning ground flashes in Sweden," 2014.

[23] C.L. Wooi, et al., "A Comparative Study on the Positive Lightning Return Stroke Electric Fields in Different Meteorological Conditions," Advances in Meteorology, vol. 2015, p. 12, 2015.

[24] N. Azlinda Ahmad, et al., "The first electric field pulse of cloud and cloud-to-ground lightning discharges," Journal of Atmospheric and Solar-Terrestrial Physics, vol. 72, pp. 143-150, 2010.

[25] N. Azlinda Ahmad, et al., "Characteristics of narrow bipolar pulses observed in Malaysia," Journal of Atmospheric and Solar-Terrestrial Physics, vol. 72, pp. 534-540, 2010.

[26] B. Salimi, et al., "Statistical analysis of lightning electric field measured under Malaysian condition," Asia-Pacific Journal of Atmospheric Sciences, vol. 50, pp. 133-137, 2014.

[27] Z.A. Baharudin, "Characterizations of ground flashes from tropic to northern region," 2014

[28] A. Nag and V.A. Rakov, "Pulse trains that are characteristic of preliminary breakdown in cloud-to-ground lightning but are not followed by return stroke pulses," Journal of Geophysical Research: Atmospheres (1984-2012), vol. $113,2008$.

[29] V. Cooray, "Further characteristics of positive radiation fields from lightning in Sweden," Journal of Geophysical Research: Atmospheres (1984-2012), vol. 89, pp. 11807-11815, 1984. 


\section{BIOGRAPHIES OF AUTHORS}

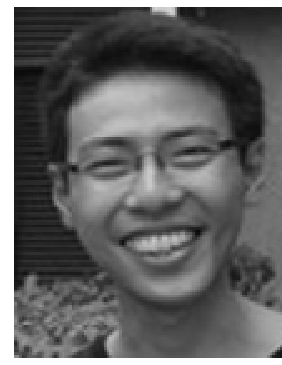

Chin-Leong Wooi received the B.Sc. degree in electrical and electronic engineering from the Universiti Malaysia Sabah, Malaysia, in 2011, the M.E. degree in electrical engineering from the Universiti Teknologi Malaysia, Johor, Malaysia, in 2013, and is currently pursuing the Ph.D. degree in electrical engineering at the Institute of High Voltage and High Current (IVAT), Universiti Teknologi Malaysia. His research interest includes the high voltage engineering, electromagnetic field and lightning measurement.

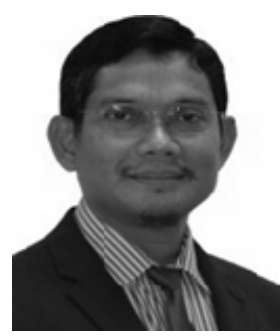

Z. Abdul-Malek received the B.E. degreein electrical and computer systems from Monash University, Melbourne, Australia, in 1989, the M.Sc.degree in electrical and electromagnetic engineering with industrial applications from the University of Wales Cardiff, Cardiff, U.K., in 1995 and the Ph.D.degree in high voltage engineering from Cardiff University, Cardiff, U.K., in 1999. He was a Lecturer with Universiti Teknologi Malaysia (UTM) for 25 years, where he is currentlya Professor of High Voltage Engineeringwith the Faculty of Electrical Engineering. He is currently the Director of theInstitute of High Voltage and High Current (IVAT), UTM. He has publishedtwo books, and has authored and co-authored more than 100 papers in varioustechnical journals and conference proceedings. His research interests includehigh-voltage instrumentation, lightning protection, detection and warning systems, partial discharges, nanodielectrics, and condition monitoring of power equipment. Dr. Abdul-Malek is actively involved in many national committees. He is the Chairman, Working Group on High-Voltage and High-Current Test Techniques. He is also a member of IEC Certification Body Management Committee, Technical Committee on High Voltage Power Transmission, Working Group on High Voltage Switchgear and Controlgears, Technical Working Group for Electrical Testing, and Department of Standards IEC 17025 Technical Assessors. He is amember of the Power and Energy Society, Dielectrics and Electrical Insulation Society, and CIGRE.

Noor Azlinda Ahmad received her B.Eng. (Electrical) and M. Eng. (Electrical) Degree from Universiti Teknologi Malaysia in 2001 and 2003, respectively. She completed her $\mathrm{PhD}$ in Atmospheric Discharge from Uppsala University, Sweden in 2011. At present she is a lecturer at Institute of High Voltage and High Current (IVAT) Universiti Teknologi Malaysia. Her major research interests are lightning protection, measurement, detection and characterization.

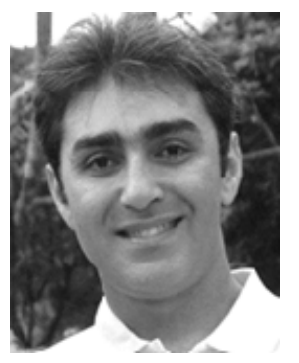

M. Mokhtari received the B.Sc. degree in electrical engineering from the University of Science and Technology, Tehran, Iran, in 2003, the M.E. degree in electrical engineering from the Universiti Teknologi Malaysia, Johor, Malaysia, in 2013, and is currently pursuing the Ph.D. degree in electrical engineering at the Institute of High Voltage and High Current (IVAT), Universiti Teknologi Malaysia. His research interest includes the high voltage engineering, the power system transient simulation, software development for high-frequency grounding systems, and insulation coordination.

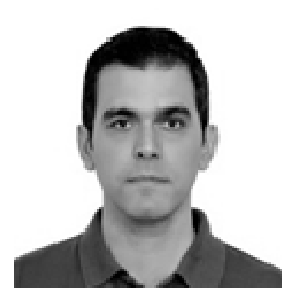

B. Salimi received the B.Sc. degree in electrical engineering from the Azad University (Saveh Branch), Iran, in 2008, the M.E. degree in electrical engineering from the Universiti Teknologi Malaysia, Johor, Malaysia, in 2011, and is currently pursuing the Ph.D. degree in electrical engineering at the Institute of High Voltage and High Current (IVAT), Universiti Teknologi Malaysia. His research interest includes the Lightning Discharges, power system transient simulation, and insulation coordination. 